[3] G. V. Moustakides and S. Theodoridis, "Fast newton transversal filters-a new class of adaptive estimation algorithms," IEEE Trans. Signal Process., vol. 39, no. 10, pp. 2184-2193, Oct. 1991.

[4] B. F. Boroujeny, "Fast LMS/newton algorithms based on autoregressive modeling and their application to acoustic echo cancellation," IEEE Trans. Signal Process., vol. 45, no. 8, pp. 1987-2000, Aug. 1997.

[5] J. Benesty, T. Gansler, D. R. Morgan, M. M. Sondhi, and S. L. Gay, Advances in Network and Acoustic Echo Cancellation. New York: Springer-Verlag, 2001.

[6] G. A. Clark, S. K. Mitra, and S. R. Parker, "Block implementation of adaptive digital filters," IEEE Trans. Acoust., Speech, Signal Process., vol. ASSP-29, no. 3, pp. 744-752, Jun. 1981.

[7] S. Narayan, A. M. Peterson, and M. J. Narasimha, "Transform domain LMS algorithm," IEEE Trans. Acoust. Speech, Signal Process., vol. ASSP-31, no. 3, pp. 609-615, Jun. 1983.

[8] J. Benesty and P. Duhamel, "A fast exact least mean square adaptive algorithm,” IEEE Trans. Signal Process., vol. 40, no. 12, pp. 2904-2920, Dec. 1992.

[9] K. Berberidis and S. Theodoridis, "A new fast block adaptive algorithm," IEEE Trans. Signal Process., vol. 47, no. 1, pp. 75-87, Jan. 1999.

[10] D. T. M. Slock and K. Maouche, "The fast subsampled-updating recursive least-square (FSU-RLS) algorithm for adaptive filtering based on displacement structure and FFT," Signal Process., vol. 40, pp. 5-20, Oct. 1994.

[11] M. Tanaka, S. Makino, and J. Kojima, "A block exact fast affine projection algorithm," IEEE Trans. Speech Audio Process., vol. 7, no. 1, pp. 79-86, Jan. 1999.

[12] G. Rombouts and M. Moonen, "A sparse block exact affine projection algorithm," IEEE Trans. Speech Audio Process., vol. 10, no. 2, pp. 100-108, Feb. 2002.

[13] F. Albu and H. K. Kwan, "Fast block exact Gauss-Seidel pseudo affine projection algorithm," IEE Electron. Lett., vol. 40, no. 22, pp. 1451-1453, Oct. 2004.

[14] Z. J. Mou and P. Duhamel, "Fast FIR filtering: Algorithms and implementation," Signal Process., vol. 377-384, Dec. 1987.

[15] Z. J. Mou and P. Duhamel, "Short-length FIR filters and their use in fast nonrecursive filtering," IEEE Trans. Signal Process., vol. 39, no. 6, pp. 1322-1332, Jul. 1991.

[16] Y. Zhou, S. C. Chan, and K. L. Ho, "A new block exact fast LMS/newton adaptive filtering algorithm," in Proc. IEEE 2004 47th Midwest Symp. Circuits Systems, Hiroshima, Japan, Jul. 25-28, 2004, pp. II-29-II-32.

[17] Digital Network Echo Cancellers, ITU-T Recommendation G.168, 2000 .

[18] K. Ikeda, S. Tanaka, and Y. Wang, "Convergence rate analysis of fast predictor-based least squares algorithm," IEEE Trans. Circuits Syst. II: Analog Digit. Signal Process., vol. 49, no. 1, pp. 11-15, Jan. 2002.

[19] Y. Wang, K. Ikeda, and K. Nakayama, "A numerically stable fast Newton-type adaptive filter based on order recursive least squares algorithm," IEEE Trans. Signal Process., vol. 51, no. 9, pp. 2357-2368, Sep. 2003.

\section{On the Spectral Factor Ambiguity of FIR Energy Compaction Filter Banks}

Andre Tkacenko and P. P. Vaidyanathan

Abstract-This paper focuses on the design of signal-adapted finite-impulse response (FIR) paraunitary (PU) filter banks optimized for energy compaction (EC). The design of such filter banks has been shown in the literature to consist of the design of an optimal FIR compaction filter followed by an appropriate Karhunen-Loève transform (KLT). Despite this elegant construction, EC optimal filter banks have been shown to perform worse than common nonadapted filter banks for coding gain, contrary to intuition. Here, it is shown that this phenomenon is most likely due to the nonuniqueness of the compaction filter in terms of its spectral factors. This nonuniqueness results in a finite set of EC optimal filter banks. By choosing the spectral factor yielding the largest coding gain, it is shown that the resulting filter bank behaves more and more like the infinite-order principal components filter bank (PCFB) in terms of numerous objectives such as coding gain, multiresolution, noise reduction with zeroth-order Wiener filters in the subbands, and power minimization for discrete multitone (DMT)-type nonredundant transmultiplexers.

Index Terms-Compaction filter, energy compaction, multirate filter bank, principal components filter bank.

\section{INTRODUCTION}

A signal-adapted filter bank is any multirate filter bank whose filters depend on the nature or statistics of its input. The problem of the design of optimal signal-adapted multirate filter banks has been of interest to the signal processing community on account of its applications in data compression, signal denoising, and digital communications [11], [15], [16]. A typical model for the filter bank used is the $M$-channel maximally decimated filter bank [14] shown in Fig. 1(a). Here, the subband processors $\left\{\mathcal{P}_{k}\right\}$ need not be linear and are typically scalar quantizers, constant multipliers, or threshold devices.

An equivalent polyphase representation [14] of the filter bank of Fig. 1(a) is shown in Fig. 1(b). The analysis filters $\left\{H_{k}(z)\right\}$ and synthesis filters $\left\{F_{k}(z)\right\}$ are, respectively, related to the analysis polyphase matrix $\mathbf{H}(z)$ and synthesis polyphase matrix $\mathbf{F}(z)$ as follows [14]:

$$
\begin{aligned}
{\left[\begin{array}{llll}
H_{0}(z) & H_{1}(z) & \cdots & H_{M-1}(z)
\end{array}\right]^{T} } & =\mathbf{H}\left(z^{M}\right) \mathbf{a}(z) \\
{\left[\begin{array}{lllll}
F_{0}(z) & F_{1}(z) & \cdots & F_{M-1}(z)
\end{array}\right] } & =\widetilde{\mathbf{a}}(z) \mathbf{F}\left(z^{M}\right) .
\end{aligned}
$$

Here, $\mathbf{a}(z)$ denotes the $M \times 1$ advance chain vector given by

$$
\mathbf{a}(z)=\left[\begin{array}{llll}
1 & z & \cdots & z^{M-1}
\end{array}\right]^{T}
$$

and the tilde notation denotes the paraconjugate [14] of any system (i.e., $\widetilde{\mathbf{A}}(z) \triangleq \mathbf{A}^{\dagger}\left(1 / z^{*}\right)$ for any $\left.\mathbf{A}(z)\right)$.

From here on, we will assume that the $M$-fold blocked signal vector $\mathbf{x}(n)$ from Fig. 1(b) is wide sense stationary (WSS) with a known

Manuscript received August 6, 2004; revised January 19, 2005. This work was supported in part by the NSF grant CCF-0428326, ONR grant N00014-06-10011, and the California Institute of Technology. The associate editor coordinating the review of this manuscript and approving it for publication was Prof. Trac D. Tran.

A. Tkacenko was with the Department of Electrical Engineering, California Institute of Technology, Pasadena, CA 91125 USA. He is now with the Digital Signal Processing Research Group, Jet Propulsion Laboratory, Pasadena, CA 91109 USA (e-mail: andre@systems.caltech.edu).

P. P. Vaidyanathan is with the Department of Electrical Engineering, California Institute of Technology, Pasadena, CA 91125 USA (e-mail: ppvnath@systems.caltech.edu).

Digital Object Identifier 10.1109/TSP.2005.861060 


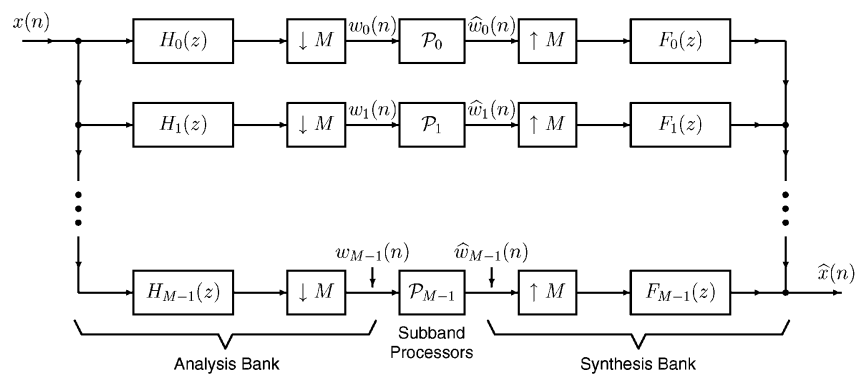

(a)

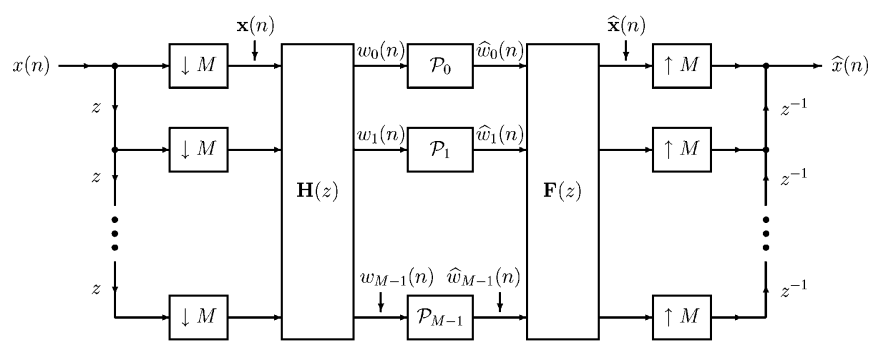

(b)

Fig. 1. (a) Typical $M$-channel maximally decimated filter bank system. (b) Polyphase implementation of filter bank.

power spectral density (psd) $\mathbf{S}_{\mathbf{x x}}(z)$. This is equivalent to saying that the scalar input signal $x(n)$ is cyclo-WSS with period $M$ (abbreviated CWSS $(M))$ [14].

We will restrict our attention to paraunitary (PU) or orthonormal filter banks in which we have

$$
\mathbf{H}(z)=\widetilde{\mathbf{F}}(z), \widetilde{\mathbf{F}}(z) \mathbf{F}(z)=\mathbf{I} \forall z .
$$

Orthonormal filter banks have been shown to be useful on account of their ease of design and energy preserving properties [14]. In fact, such filter banks are used to generate wavelet bases [14] and have even been used for wavelet-based lossy data compression such as that used by JPEG 2000 [10].

\section{A. Principal Components Filter Bank}

Recently, it has been shown that a special type of PU filter bank known as the principal components filter bank (PCFB) [11], if it exists for a particular filter bank class, is simultaneously optimal for a variety of data-compression-type objectives [1], including mean-squared error under the presence of quantization noise [5] (for any bit allocation) and coding gain [15] (with optimal bit allocation). By definition, a PCFB for an input psd $\mathbf{S}_{\mathbf{x x}}(z)$ and a class $\mathcal{C}$ of filter banks, if it exists, is one whose subband variance vector

$$
\boldsymbol{\sigma} \triangleq\left[\begin{array}{llll}
\sigma_{w_{0}}^{2} & \sigma_{w_{1}}^{2} & \cdots & \sigma_{w_{M-1}}^{2}
\end{array}\right]^{T}
$$

majorizes [2] any other subband variance vector arising from any other filter bank from $\mathcal{C}$. (Recall that a vector $\mathbf{a} \triangleq\left[\begin{array}{llll}a_{0} & a_{1} & \cdots & a_{P-1}\end{array}\right]^{T}$ with $a_{0} \geq a_{1} \geq \cdots \geq a_{P-1} \geq 0$ is said to majorize [2] a vector $\mathbf{b} \triangleq$ $\left[b_{0} b_{1} \cdots b_{P-1}\right]^{T}$ with $b_{0} \geq b_{1} \geq \cdots \geq b_{P-1} \geq 0$ iff we have

$$
\left.\sum_{k=0}^{p} a_{k} \geq \sum_{k=0}^{p} b_{k} \forall 0 \leq p \leq P-2, \sum_{k=0}^{P-1} a_{k}=\sum_{k=0}^{P-1} b_{k} .\right)
$$

In addition to being optimal for coding gain and mean-squared error in the presence of quantization noise, the PCFB has also been shown to be optimal for any concave objective function of $\boldsymbol{\sigma}$ [1].

1) Classes for Which PCFB's Exist: For general input power spectra, PCFBs are only known to exist for certain classes of filter banks. One notable exception is special case where $M=2$, in which case a PCFB always exists for any class of PU filter banks [1]. For general $M$, however, PCFBs are known to exist only for two special classes. If $\mathcal{C}$ is the class of all transform coders $\mathcal{C}^{t}$, in which $\mathbf{F}(z)$ is a constant unitary matrix $\mathbf{T}$, then the PCFB exists and is the Karhunen-Loève transform (KLT) for $\mathbf{x}(n)$ (i.e., $\mathbf{T}$ diagonalizes the autocorrelation matrix $\mathbf{R}_{\mathbf{x x}}(0)$ ) [1], [3]. Furthermore, if $\mathcal{C}$ is the class of all (unconstrained order) PU filter banks $\mathcal{C}^{u}$, then the PCFB exists and is the pointwise in frequency $\operatorname{KLT}$ for $\mathbf{x}(n)$ [1], [15], [16]. By this, we mean that $\mathbf{F}\left(e^{j \omega}\right)$ diagonalizes (i.e., totally decorrelates) $\mathbf{S}_{\mathbf{x x}}\left(e^{j \omega}\right)$ for every $\omega$ such that the frequency-dependent eigenvalues are always arranged in decreasing order, which is a property called spectral majorization [15]. For many practical cases of inputs (for example, if the scalar input $x(n)$ is itself WSS), the corresponding analysis and synthesis filters are ideal bandpass filters called compaction filters [13], [15]. As such, they are infinite order and, hence, unrealizable in practice and serve only to compute an upper bound on the performance that we can expect from a PU filter bank.

2) Problems With the Class of FIR PU Filter Banks: For the class of FIR PU filter banks in which $\mathbf{F}(z)$ has a certain finite McMillan degree [14], it is believed that a PCFB does not exist [1], [4], [6] although this has not yet been formally proven. Instead, for this class, $\mathbf{F}(z)$ is typically chosen to optimize a specific objective such as coding gain [7], [18] or rate distortion [8]. All such methods require the numerical optimization of nonconvex functions which offer little insight into the behavior of the solutions as the filter order or degree of $\mathbf{F}(z)$ increases. Although we might expect these filter banks to behave increasingly like the infinite-order PCFB as we increase the order, this has not been shown in the literature.

\section{B. Main Contributions of Paper}

In this paper, we focus on the design of FIR PU filter banks optimized for an energy compaction (EC) criterion originally considered in [9]. This elegant method only requires the computation of an FIR compaction filter followed by an appropriate KLT as we review in Section II-C. Although such filter banks were shown to perform poorly in terms of coding gain in [9], contrary to intuition, we show here that this is most likely due to the nonuniqueness of the compaction filter in terms of its spectral factors. This nonuniqueness leads to a finite set of EC optimal filter banks. By choosing the spectral factor yielding the largest coding gain, we show that there are numerous benefits to be reaped. In particular, we show that this solution exhibits a tendency toward the infinite-order PCFB as the filter order is increased. This tendency is shown in terms of coding gain, multiresolution, noise reduction with zeroth-order Wiener filters in the subbands, and power minimization for discrete multitone (DMT)-type nonredundant transmultiplexers. Such PCFB-like behavior has not previously been reported in the literature.

\section{The ENERgy COMPACTION CRITERION}

Referring to Fig. 1(a), in [9], Moulin and Mihçak opted to design an FIR PU signal-adapted filter bank according to the following EC criterion.

EC Criterion:

1) Maximize $\sigma_{w_{0}}^{2}$ subject to

$\left[\widetilde{F}_{0}(z) F_{0}(z)\right]_{\downarrow M}=1 \quad$ (Compaction Filter Problem). 
2) For $i=1,2, \ldots, M-1$ successively maximize $\sigma_{w_{i}}^{2}$ subject to $\left[\widetilde{F}_{i}(z) F_{i}(z)\right]_{\rfloor M}=1 \quad($ Nyquist $(M)$ Criterion $)$ $\left[\widetilde{F}_{k}(z) F_{i}(z)\right]_{\downarrow M}=0 \forall 0 \leq k \leq i-1 \quad$ (Orthogonality Criterion).

The PCFB, if it exists for a particular filter bank class, is optimal for energy compaction [1], [11]. For the FIR class, it can be shown that using the complete parameterization of FIR PU systems in terms of Householder-like building blocks [14], [17], which we review in Section II-B, the entire filter bank can be completed via an appropriate KLT once an optimal compaction filter has been found (Step 1 from above). This is shown in Section II-C. In the next section, we briefly review the compaction filter problem.

\section{A. Compaction Filter Problem}

For the special case in which the input $x(n)$ is WSS with psd $S_{x x}\left(e^{j \omega}\right)$, which we will henceforth assume to be the case, the compaction filter problem (Step 1 of the EC criterion) becomes the following [15]:

$$
\begin{aligned}
& \text { Maximize } \sigma_{w_{0}}^{2}=\frac{1}{2 \pi} \int_{0}^{2 \pi} S_{x x}\left(e^{j \omega}\right)\left|F_{0}\left(e^{j \omega}\right)\right|^{2} d \omega \\
& \text { subject to }\left[\left|F_{0}\left(e^{j \omega}\right)\right|^{2}\right]_{\downarrow M}=1 \forall \omega .
\end{aligned}
$$

This problem is only a function of the magnitude of $F_{0}\left(e^{j \omega}\right)$ and not on its phase. As such, the solution to (3) is not unique. In particular, any spectral factor of a given optimal $F_{0}\left(e^{j \omega}\right)$ is itself an optimal solution.

If no order constraint is made on $F_{0}(z)$, the optimal compaction filter has an ideal bandpass response that depends on $S_{x x}\left(e^{j \omega}\right)$ [15]. For the unconstrained order PCFB, the analysis/synthesis filters are ideal compaction filters for $S_{x x}\left(e^{j \omega}\right)$ and its peeled spectra [15]. As such, these filters are infinite order and unrealizable. This is why the unconstrained-order PCFB is often referred to as the infinite-order PCFB.

If we impose an FIR constraint on $F_{0}(z)$, the design of an optimal compaction filter becomes more complicated and must be done numerically. In [12], Tuqan and Vaidyanathan proposed a semidefinite programming (SDP) method based on a state-space description of the compaction filter that was shown to be globally optimal. It is for this reason that this method was used for all of the simulations presented here.

\section{B. Complete Parameterization of FIR PU Systems}

Once Step 1 of the EC criterion (the FIR compaction filter problem) is completed, Step 2 can be easily carried out upon exploiting the complete parameterization of the FIR PU synthesis polyphase matrix in terms of Householder-like building blocks [14], [17]. Suppose that $\mathbf{F}(z)$ is a $p \times r$ causal FIR system with $p \geq r$. Then, $\mathbf{F}(z)$ is PU with McMillan degree $(N-1)$ iff it can be expressed as [14], [17]

$$
\mathbf{F}(z)=\underbrace{\mathbf{V}_{N-1}(z) \mathbf{V}_{N-2}(z) \cdots \mathbf{V}_{1}(z)}_{\mathbf{V}(z)} \mathbf{U}
$$

where $\mathbf{U}$ is a unitary $p \times r$ matrix and $\mathbf{V}_{k}(z)$ is a $p \times p$ Householder-like PU degree-one system with

$$
\mathbf{V}_{k}(z)=\mathbf{I}_{p}-\mathbf{v}_{k} \mathbf{v}_{k}^{\dagger}+z^{-1} \mathbf{v}_{k} \mathbf{v}_{k}^{\dagger}, 1 \leq k \leq N-1
$$

and $\mathbf{v}_{k}$ is a $p \times 1$ unit norm vector for all $k$.

In general, the matrix $\mathbf{U}$ from (4) is unique [14]. When $r>1$, the vectors $\mathbf{v}_{k}$ from (5) are in general not unique. In contrast to this, when $r=1$, i.e., when $\mathbf{F}(z)$ is a vector system, the diadic terms $\mathbf{v}_{k} \mathbf{v}_{k}^{\dagger}$ appearing in (5) are unique [14], [17]. This uniqueness in the vector case, as we will soon show, greatly facilitates the design of an EC optimal FIR filter bank once a suitable compaction filter has been designed.

\section{Simplifying the EC Criterion for FIR PU Filter Banks}

Suppose that Step 1 of the EC criterion has been completed. In other words, suppose a causal FIR compaction filter $F_{0}(z)$ of length $M N$ has been computed. Then, this is equivalent to saying that we know $\mathbf{f}_{0}(z)$, the first column of $\mathbf{F}(z)$. This is because, from (1), we have [14]

$$
\begin{aligned}
F_{0}(z) & =\widetilde{\mathbf{a}}(z) \mathbf{f}_{0}\left(z^{M}\right) \Longleftrightarrow\left[\mathbf{f}_{0}(z)\right]_{k} \\
& =\left[z^{k} F_{0}(z)\right]_{\downarrow M}, \quad 0 \leq k \leq M-1 .
\end{aligned}
$$

However, recall now that $\mathbf{F}(z)$ is an FIR PU system of the form as shown in (4). Thus, we have

$$
\mathbf{f}_{0}(z)=\mathbf{V}(z) \mathbf{u}_{0}
$$

where $\mathbf{u}_{0}$ is the first column of the matrix $\mathbf{U}$ given in (4). Hence, (6) is a Householder-like parameterization of the FIR PU vector system $\mathbf{f}_{0}(z)$ and so the matrix $\mathbf{V}(z)$ from (6) is unique. In other words, once $F_{0}(z)$ has been found, we can uniquely determine the matrix $\mathbf{V}(z)$ appearing in the parameterization of $\mathbf{F}(z)$. Furthermore, we know the first column of the matrix $\mathbf{U}$ appearing in this parameterization.

To obtain the remaining degrees of freedom, from Step 2 of the EC criterion, it can be easily shown [9] that the matrix $\mathbf{U}$ can be obtained by computing the KLT corresponding to the psd $\widetilde{\mathbf{V}}(z) \mathbf{S}_{\mathbf{x x}}(z) \mathbf{V}(z)$. Hence, the entire filter bank can be easily completed once a suitable compaction filter has been computed.

To summarize, with an FIR constraint in effect, an EC optimal filter bank can be constructed as follows.

Computing An FIR EC Optimal Filter Bank:

1) Calculate an optimal FIR compaction filter $F_{0}(z)$.

2) Obtain the unique matrix $\mathbf{V}(z)$ corresponding to the vector of polyphase components of $F_{0}(z)$.

3) Compute the matrix $\mathbf{U}$ to be the KLT for the process with psd $\widetilde{\mathbf{V}}(z) \mathbf{S}_{\mathbf{x x}}(z) \mathbf{V}(z)$.

Through simulations, we will show that different spectral factors of a given compaction filter yield the same performance for Step 1 of the EC criterion but varying performance for Step 2, as we might expect.

\section{Simulation Results For EC DESIGNEd FIR PU Filter BANKS}

In [9], only the minimum-phase compaction filter was used to construct an EC optimal filter bank. There, despite the use of a nearly optimal compaction filter, the resulting filter banks performed poorly in terms of coding gain, contrary to intuition. Here, by evaluating the performance of all spectral factors and choosing the one yielding the largest coding gain, we can obtain a filter bank with a significant improvement in coding gain. Furthermore, this filter bank exhibits an increasingly PCFB-like behavior in terms of numerous objectives as we increase the order, as shown here. Although this exhaustive search implies an increase in complexity that is exponential in the filter order, this is often mitigated by the fact that we may only seek a real coefficient compaction filter and that an optimal compaction filter consists of many unit circle zeros.

For all simulations, the input $x(n)$ was chosen to be a real autoregressive order $4(\mathrm{AR}(4))$ process whose psd $S_{x x}\left(e^{j \omega}\right)$ is shown in Fig. 2. We opted to design an $M=4$ channel system. The synthesis polyphase matrix order $N$ was varied from 1 to 10 in order to gauge the behavior 


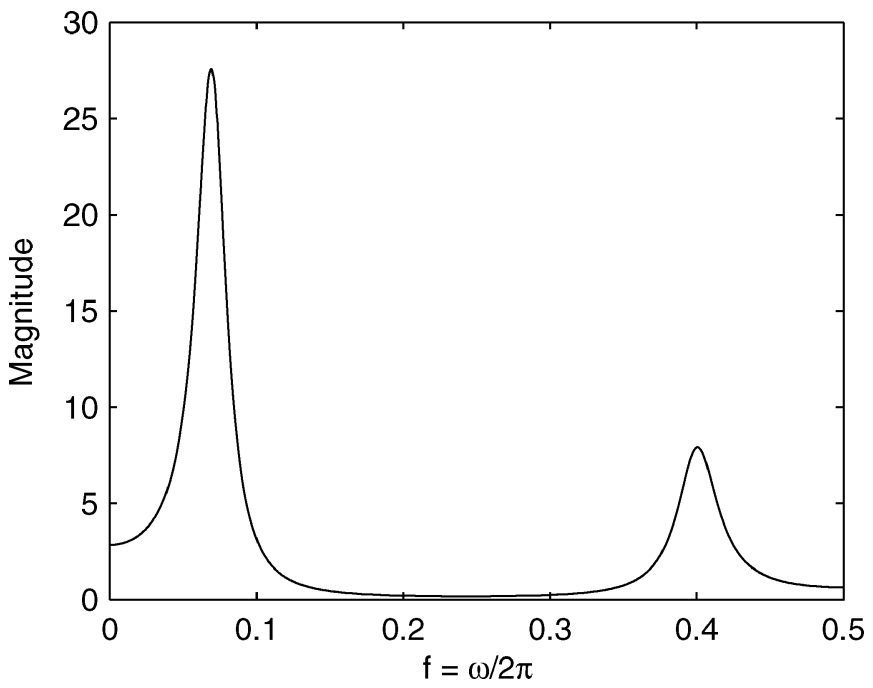

Fig. 2. Input psd $S_{x x}\left(e^{j \omega}\right)$ of the $\mathrm{AR}(4)$ process $x(n)$.

of the filter banks designed. For the design of the FIR compaction filter, the globally optimal SDP method of [12] was used.

\section{A. Coding Gain Results}

Recall that for any PU filter bank with optimal bit allocation, the coding gain is given by [14]

$$
G_{\text {code }}=\frac{\left(\frac{1}{M} \sum_{k=0}^{M-1} \sigma_{w_{k}}^{2}\right)}{\left(\prod_{k=0}^{M-1} \sigma_{w_{k}}^{2}\right)^{\frac{1}{M}}} .
$$

In Fig. 3, we have plotted the observed coding gain as a function of $N$ for the minimum-phase and coding gain optimized EC filter banks. Also included is the coding gain of the KLT and infinite-order PCFB. As can be seen, the coding gain optimized filter bank exhibited a noticeable improvement over the one yielded by the minimum-phase spectral factor. Furthermore, the coding gain optimized EC filter bank exhibited a monotonically increasing coding gain, as opposed to that of the minimum-phase spectral factor.

The zero locations of the optimal compaction filter spectral factor are shown in Fig. 4. Clearly, this factor is not minimum phase. Alhough the total number of spectral factors to check is $2^{M N-1}$, many can be ignored due to unit circle zeros and complex coefficient solutions. From Fig. 4 , it is clear that for the case $N=10$, of the $2^{39}$ possible spectral factors, only $2^{5}=32$ distinct real coefficient factors exist.

It should be noted from Fig. 3 that even though the optimized filter bank exhibited a monotonic increase in coding gain with order, the gain was always well below that of the infinite-order PCFB. This is most likely due to the stringent ideal bandpass requirements of the PCFB filters. In Figs. 5 and 6, we have plotted, respectively, the magnitude-squared responses of the synthesis filters corresponding to the minimum-phase and coding gain optimal spectral factors for $N=10$. As can be seen, with the exception of the compaction filter $F_{0}(z)$, the FIR filters represent poor approximations to the PCFB ones. For the optimized filter bank, the second filter $F_{1}(z)$ comes close to the performance of that of the PCFB, but to a lesser extent than the FIR compaction filter. This suggests that the EC criterion, which places nearly all of the design emphasis on the first filter $F_{0}(z)$, may be too restrictive to yield an adequate coding gain.

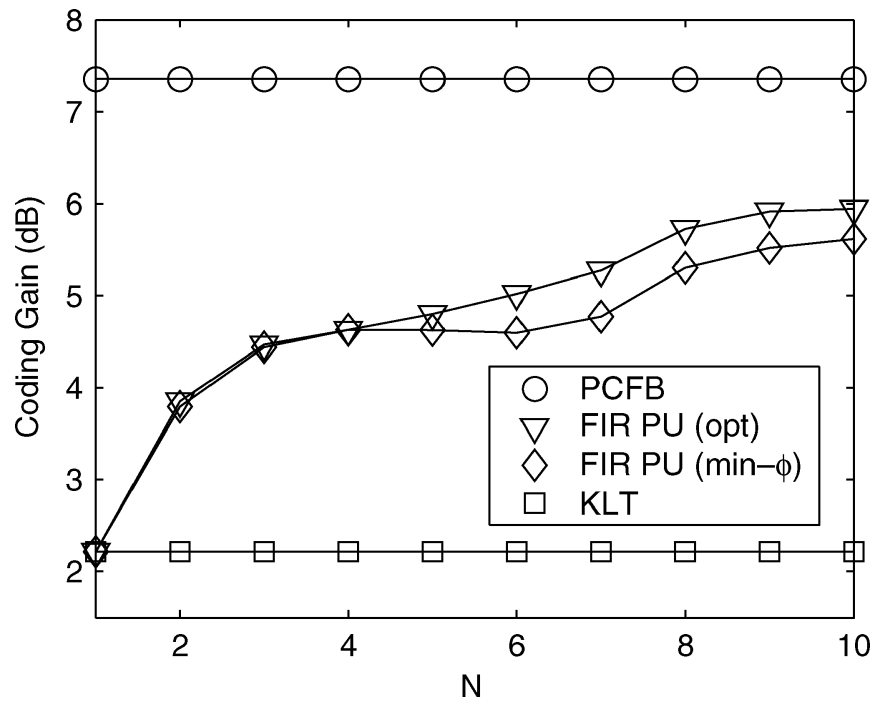

Fig. 3. Observed coding gain $G_{\text {code }}$ as a function of the synthesis polyphase order $N$.

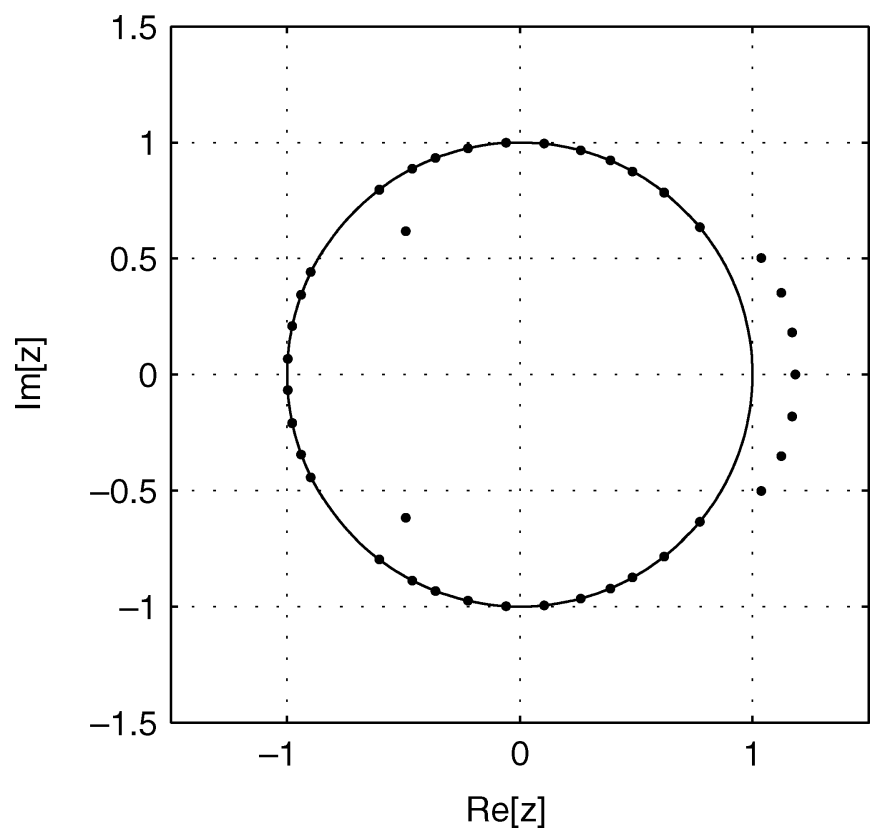

Fig. 4. Zero locations of the optimal spectral factor for $N=10(M N-1=$ 39).

\section{B. Multiresolution Optimality Results}

One measure of multiresolution optimality is the proportion of partial subband variances to the total. By preserving only $L$ out of $M$ subbands, this quantity is given by

$$
P(L)=\frac{\left(\sum_{k=0}^{L-1} \sigma_{w_{k}}^{2}\right)}{\left(\sum_{k=0}^{M-1} \sigma_{w_{k}}^{2}\right)}, \quad 1 \leq L \leq M .
$$

The PCFB, if it exists, maximizes $P(L)$ for all $L$ due to its majorization property (see Section I-A).

In Fig. 7, we have plotted the observed $P(L)$ versus $L$ for the previously designed EC filter banks for (a) $N=2$ and (b) $N=10$. As we increase $N$ from 2 to 10, it can be seen that both FIR EC filter banks are trying to emulate the behavior of the infinite order PCFB, 


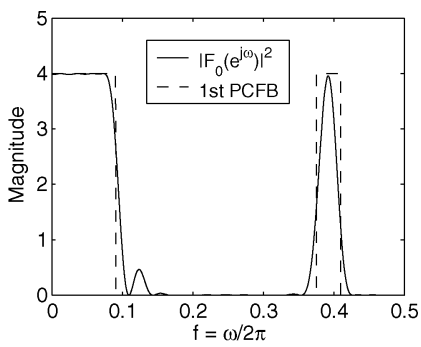

(a)

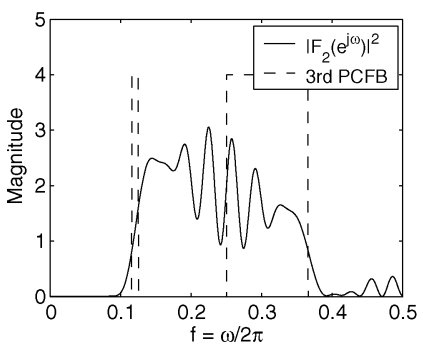

(c)

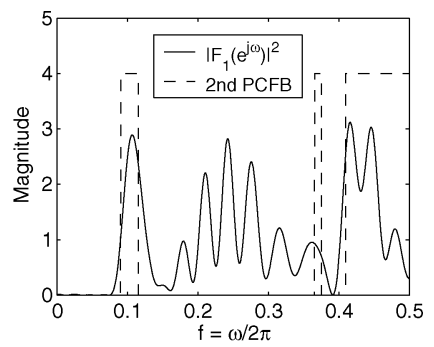

(b)

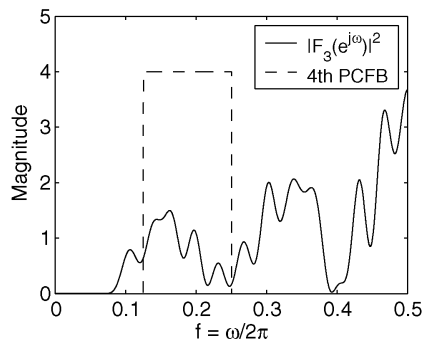

(d)
Fig. 5. Synthesis filter magnitude- squared responses for the minimum-phase spectral factor for $N=10$. (a) First channel. (b) Second channel. (c) Third channel. (d) Fourth channel.

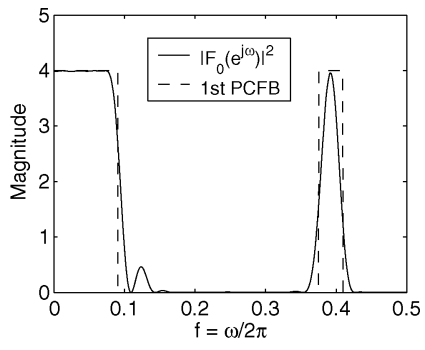

(a)

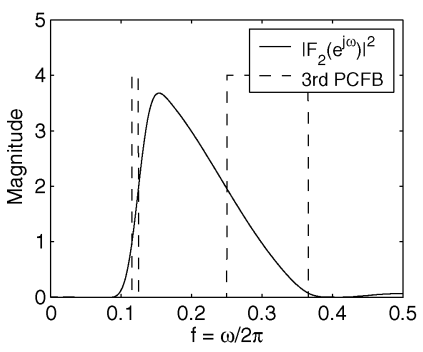

(c)

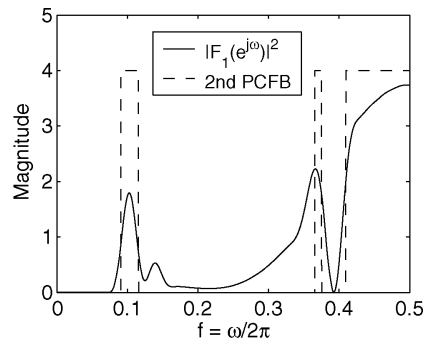

(b)

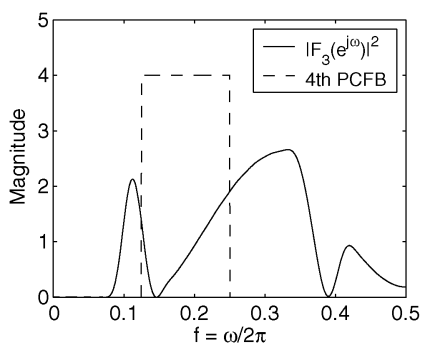

(d)
Fig. 6. Synthesis filter magnitude-squared responses for the coding gain optimal spectral factor for $N=10$. (a) First channel. (b) Second channel. (c) Third channel. (d) Fourth channel.

in line with intuition. Although both FIR filter banks exhibit similar performance, the coding gain optimized one always performed at least as well as the minimum-phase one (expect for a slight discrepancy at $L=3$ for $N=2$ ). As can be seen, the FIR filter banks appear to converge slowly to the PCFB limit for $L=2$. This is probably due to the multiple narrow passband regions of the second PCFB filter as can be seen in Figs. 5(b) and 6(b).

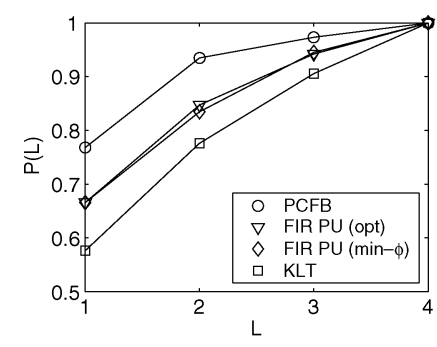

(a)

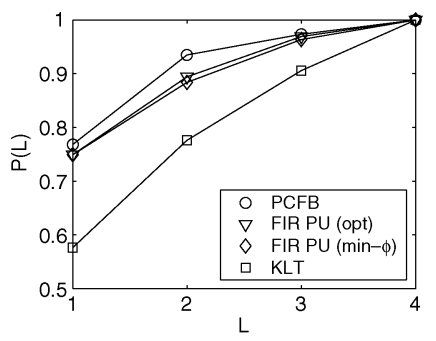

(b)
Fig. 7. Proportion $P(L)$ of the total variance as a function of the number of subbands kept $L$ for (a) $N=2$ and (b) $N=10$.

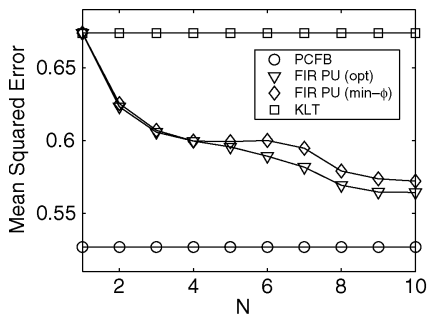

(a)

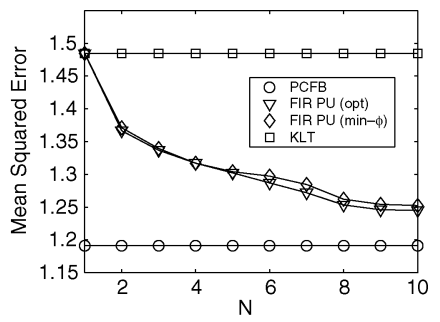

(b)
Fig. 8. Mean-squared error with zeroth-order subband Wiener filters $\xi$ as a function of $N$ for (a) $\eta^{2}=1$ and (b) $\eta^{2}=4$.

\section{Noise Reduction Using Zeroth-Order Wiener Filters}

Suppose that the input to the filter bank of Fig. 1 is $x(n)=s(n)+$ $\mu(n)$, where $s(n)$ is a pure CWSS $(M)$ signal and $\mu(n)$ is a white noise process with variance $\eta^{2}$. If the subband processors $\left\{\mathcal{P}_{k}\right\}$ are zerothorder Wiener filters [14], the mean-squared error $\xi$ of the filter bank is given by [1]

$$
\xi=\frac{1}{M} \sum_{k=0}^{M-1} \frac{\sigma_{w_{k}}^{2} \eta^{2}}{\sigma_{w_{k}}^{2}+\eta^{2}}
$$

where $\sigma_{w_{k}}^{2}$ is the $k$ th subband variance of the desired signal $s(n)$. As $\xi$ is a concave function of the subband variance vector $\sigma$ from (2), the PCFB for $s(n)$, if it exists, is optimal for minimizing $\xi$ [1].

In Fig. 8, we have plotted the observed error $\xi$ as a function of the filter order parameter $N$ for the previously designed filter banks for a noise variance of (a) $\eta^{2}=1$ and (b) $\eta^{2}=4$. As expected, the FIR filter banks come closer to the infinite-order PCFB performance as we increase the order. Here, the coding gain optimized EC filter bank always matched or exceeded the performance of the minimum-phase solution.

\section{Power Minimization for DMT-Type Nonredundant Transmultiplexers}

In addition to data compression, the theory of PCFBs has been found useful for digital communications. To see this, consider the DMT-type transmultiplexer shown in Fig. 9. If the noise $\eta(n)$ is Gaussian and the $k$ th input $x_{k}(n)$ consists of independent identically distributed (i.i.d.) pulse-amplitude-modulated (PAM) symbols with $b_{k}$ bits and power $P_{k}$, the symbol error probability in detecting $x_{k}(n)$ is given by [16]

$$
\mathcal{P}_{e}(k)=2\left(1-2^{-b_{k}}\right) \mathcal{Q}\left(\sqrt{\frac{3 P_{k}}{\left(2^{2 b_{k}}-1\right) \sigma_{q_{k}}^{2}}}\right) .
$$




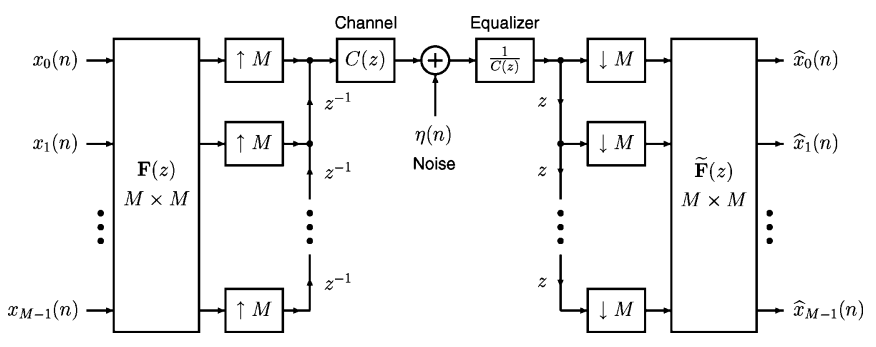

Fig. 9. Uniform PU nonredundant DMT-type transmultiplexer with a zero-forcing equalizer (ZFE) implemented.

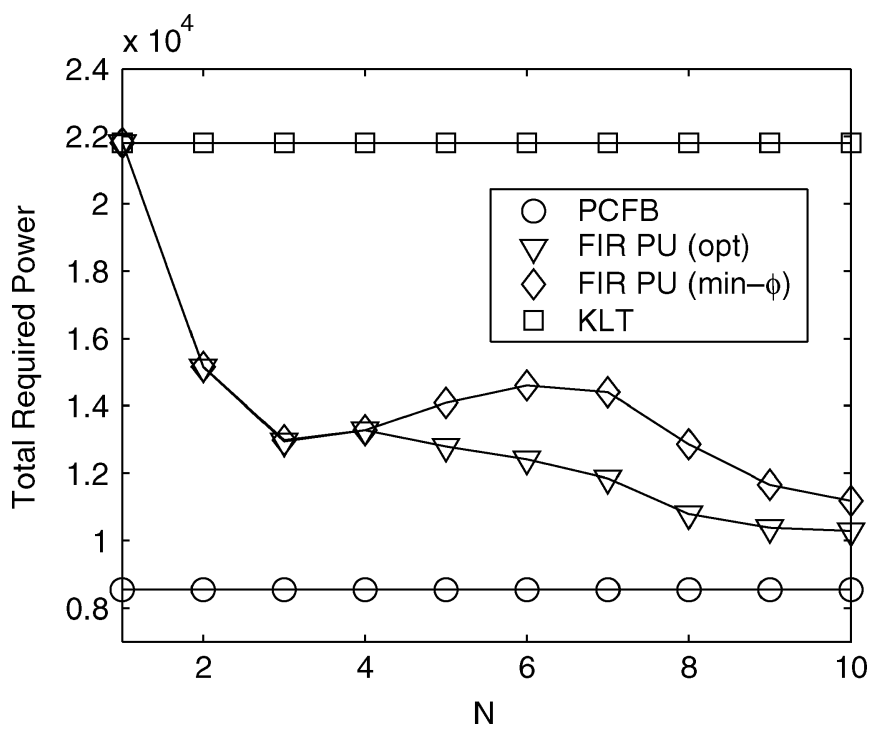

Fig. 10. Total required power $P$ for the nonredundant DMT-type transmultiplexer as a function of the order parameter $N$.

Here, $\mathcal{Q}(x)$ is the Marcum $\mathcal{Q}$ function [16], and $\sigma_{q_{k}}^{2}$ denotes the noise power seen at the $k$ th output $\widehat{x}_{k}(n)$. Solving (7) for $P_{k}$, we have that $P_{k}=\beta\left(\mathcal{P}_{e}(k), b_{k}\right) \sigma_{q_{k}}^{2}$, where $\beta\left(\mathcal{P}_{e}(k), b_{k}\right)$ is a quantity that does not depend on $\sigma_{q_{k}}^{2}$. Hence, the total required power $P$ is given by

$$
P=\sum_{k=0}^{M-1} P_{k}=\sum_{k=0}^{M-1} \beta\left(\mathcal{P}_{e}(k), b_{k}\right) \sigma_{q_{k}}^{2}
$$

which is a convex (and concave) function of $\left\{\sigma_{q_{k}}^{2}\right\}$. Hence, $P$ is minimized iff $\mathbf{F}(z)$ is a PCFB for the effective noise process seen at the receiver. If $\eta(n)$ has psd $S_{\eta \eta}\left(e^{j \omega}\right)$, the effective noise has psd $\left(S_{\eta \eta}\left(e^{j \omega}\right) /\left|C\left(e^{j \omega}\right)\right|^{2}\right)$.

As an example, suppose the effective noise psd is simply $S_{x x}\left(e^{j \omega}\right)$ from Fig. 2. In addition, suppose $\mathcal{P}_{e}(k)=10^{-9}$ for all $k$ and $b_{0}=2$, $b_{1}=3, b_{2}=4$, and $b_{3}=5$. (This is not an optimal bit allocation and only chosen as such for simplicity.) Then, the total power $P$ using the designed filter banks is shown in Fig. 10 as a function of the order parameter $N$. As can be seen, both FIR EC-designed filter banks approach the power of the PCFB but not monotonically. Despite this, the coding gain optimized spectral factor always performed at least as well as the minimum-phase solution and often noticeably better. The coding gain optimized solution appears to asymptotically approach the performance of the PCFB, in line with intuition.

\section{CONCLUDING REMARKS}

In this paper, we showed via simulations that the nonuniqueness of the compaction filter in terms of its spectral factors led to different EC optimal FIR filter banks yielding varying performances in terms of numerous objectives. For the filter banks designed (coding gain optimized and minimum phase), we showed an increased tendency toward the infinite-order PCFB as the filter order was increased. However, this tendency was shown to behave slowly for many important objectives such as coding gain and multiresolution. This suggests that the EC criterion, despite its elegant simplicity, is not well suited for these objectives, perhaps due to its predominant focus on the design of the first filter only.

\section{REFERENCES}

[1] S. Akkarakaran and P. P. Vaidyanathan, "Filterbank optimization with convex objectives and the optimality of principal component forms," IEEE Trans. Signal Process., vol. 49, no. 1, pp. 100-114, Jan. 2001.

[2] R. A. Horn and C. R. Johnson, Matrix Analysis. Cambridge, U.K.: Cambridge Univ. Press, 1985.

[3] Y. Huang and P. M. Schultheiss, "Block quantization of correlated Gaussian random variables," IEEE Trans. Commun. Syst., vol. C-10, no. 3, pp. 289-296, Sep. 1963.

[4] O. S. Jahromi, B. A. Francis, and R. H. Kwong, "Algebraic theory of optimal filterbanks," IEEE Trans. Signal Process., vol. 51, no. 2, pp. 442-457, Feb. 2003.

[5] A. Kıraç and P. P. Vaidyanathan, "Optimality of orthonormal transforms for subband coding," presented at the IEEE DSP Workshop, Bryce Canyon, UT, Aug. 1998.

[6] - "On existence of FIR principal component filter banks," in Proc. IEEE Int. Conf. Acoustics, Speech, Signal Processing, vol. 3, Seattle, WA, May 1998, pp. 1329-1332.

[7] P. Moulin, M. Anitescu, K. O. Kortanek, and F. A. Potra, "The role of linear semi-infinite programming in signal-adapted QMF bank design," IEEE Trans. Signal Process., vol. 45, no. 9, pp. 2160-2174, Sep. 1997.

[8] P. Moulin, M. Anitescu, and K. Ramchandran, "Theory of rate-distortion-optimal, constrained filterbanks-application to IIR and FIR biorthogonal designs," IEEE Trans. Signal Process., vol. 48, no. 4, pp. 1120-1132, Apr. 2000.

[9] P. Moulin and M. K. Mihçak, "Theory and design of signal-adapted FIR paraunitary filter banks," IEEE Trans. Signal Process., vol. 46, no. 4, pp. 920-929, Apr. 1998.

[10] K. Sayood, Introduction to Data Compression, 2nd ed. San Diego, CA: Academic, 2000.

[11] M. K. Tsatsanis and G. B. Giannakis, "Principal component filter banks for optimal multiresolution analysis," IEEE Trans. Signal Process., vol. 43, no. 8, pp. 1766-1777, Aug. 1995.

[12] J. Tuqan and P. P. Vaidyanathan, "A state space approach to the design of globally optimal FIR energy compaction filters," IEEE Trans. Signal Process., vol. 48, no. 10, pp. 2822-2838, Oct. 2000.

[13] M. Unser, "On the optimality of ideal filters for pyramid and wavelet signal approximation," IEEE Trans. Signal Process., vol. 41, no. 12, pp. 3591-3596, Dec. 1993.

[14] P. P. Vaidyanathan, Multirate Systems and Filter Banks. Englewood Cliffs, NJ: Prentice-Hall, 1993.

[15] - "Theory of optimal orthonormal subband coders," IEEE Trans. Signal Process., vol. 46, no. 6, pp. 1528-1543, Jun. 1998.

[16] P. P. Vaidyanathan and S. Akkarakaran, "A review of the theory and applications of optimal subband and transform coders," J. Appl. Comput. Harmonic Anal., vol. 10, pp. 254-289, 2001.

[17] P. P. Vaidyanathan, T. Q. Nguyen, Z. Doğanata, and T. Saramäki, "Improved technique for design of perfect reconstruction FIR QMF banks with lossless polyphase matrices," IEEE Trans. Acoustics, Speech, Signal Processing, vol. 37, no. 7, pp. 1042-1056, Jul. 1989.

[18] B. Xuan and R. H. Bamberger, "FIR principal component filter banks," IEEE Trans. Signal Process., vol. 46, no. 4, pp. 930-940, Apr. 1998. 